\title{
Assessment of the Dual Role of Clumping Factor A in S. Aureus Adhesion to Endothelium in Absence and Presence of Plasma
}

\author{
Jorien Claes ${ }^{1,2}$ Bartosz Ditkowski ${ }^{1, *}$ Laurens Liesenborghs ${ }^{2}$ Tiago Rafael Veloso ${ }^{1}$ Jose M. Entenza ${ }^{3}$ \\ Philippe Moreillon $^{3}$ Thomas Vanassche ${ }^{2}$ Peter Verhamme ${ }^{2}$ Marc F. Hoylaerts ${ }^{2}$ Ruth Heying ${ }^{1}$
}

${ }^{1}$ Division of Cardiovascular Developmental Biology, Department of Cardiovascular Sciences, KU Leuven, Leuven, Belgium

2 Department of Cardiovascular Sciences, Center for Molecular and Vascular Biology, KU Leuven, Leuven, Belgium

${ }^{3}$ Department of Fundamental Microbiology, University of Lausanne, Lausanne, Switzerland

Thromb Haemost

\author{
Address for correspondence Ruth Heying, MD, PhD, Division of \\ Cardiovascular Development Biology, Department of Cardiovascular \\ Sciences, KU Leuven, Box 911, Herestraat 49, 3000 Leuven, Belgium \\ (e-mail: ruth.heying@uzleuven.be).
}

\begin{abstract}
Keywords

- infective endocarditis

- clumping factor $\mathrm{A}$

- fibronectin binding proteins

- S. aureus

- von Willebrand factor

- fibrinogen

- fibronectin

Adhesion of Staphylococcus aureus to endothelial cells (ECs) is paramount in infective endocarditis. Bacterial proteins such as clumping factor $\mathrm{A}$ (ClfA) and fibronectin binding protein $A(F n b p A)$ mediate adhesion to EC surface molecules and (sub)endothelial matrix proteins including fibrinogen $(\mathrm{Fg})$, fibrin, fibronectin $(\mathrm{Fn})$ and von Willebrand factor (vWF). We studied the influence of shear flow and plasma on the binding of ClfA and FnbpA (including its sub-domains $A, A^{16+}, A B C, C D$ ) to coverslip-coated vWF, Fg/fibrin, Fn or confluent ECs, making use of Lactococcus lactis, expressing these adhesins heterologously. Global adherence profiles were similar in static and flow conditions. In the absence of plasma, L. lactis-clfA binding to $\mathrm{Fg}$ increased with shear forces, whereas binding to fibrin did not. The degree of adhesion of L. lactis-fnbpA to EC-bound Fn and of L. lactis-clfA to EC-bound $\mathrm{Fg}$, furthermore, was similar to that of $L$. lactis-clfA to coated VWF domain $A 1$, in the presence of vWF-binding protein ( $\mathrm{VWbp}$ ). Yet, in plasma, L. lactis-clfA adherence to activated EC-vWF/vWbp dropped over 10 minutes by $80 \%$ due to vWF-hydrolysis by a disintegrin and metalloproteinase with thrombospondin type 1 motif, member 13 and that of $L$. lactis-fnbpA likewise by $>70 \%$ compared to the adhesion in absence of plasma. In contrast, plasma Fg supported high L. lactis-clfA binding to resting and activated ECs. Or, in plasma $S$. aureus adhesion to active endothelium occurs mainly via two complementary pathways: a rapid but short-lived vWF/vWbp pathway and a stable integrin-coupled Fgpathway. Hence, the pharmacological inhibition of ClfA-Fg interactions may constitute a valuable additive treatment in infective endocarditis.
\end{abstract}

\section{Introduction}

Staphylococcus aureus (S. aureus) is the pathogen most frequently associated with heart valve and endovascular

\footnotetext{
* Bartosz Ditkowski performed all the experimental work during the revision and drafted the revised manuscript.
}

infections, such as infective endocarditis (IE). ${ }^{1}$ In order to cause these infections, S. aureus has developed specific mechanisms to adhere to endothelial cells (ECs) lining the heart and vessel wall and to the exposed sub-endothelial matrix. To this end, $S$. aureus expresses a large number of surface-bound and secreted proteins, contributing to the received

December 15, 2017

accepted after revision

May 2, 2018
Copyright $\odot$ Schattauer

DOI https://doi.org/ 10.1055/s-0038-1660435. ISSN 0340-6245. 
pathogenesis of endovascular infections. Various staphylococcal surface proteins or microbial surface components recognizing adhesive matrix molecules (MSCRAMMs) have been extensively described as mediators of adhesion to host cells and to extracellular matrix proteins, e.g. fibronectin (Fn)-binding protein $\mathrm{A}$ (FnbpA), clumping factor $\mathrm{A}$ (ClfA) and staphylococcal protein $A(S p A) .{ }^{2-11}$ These MSCRAMMs interact with Fn, fibrinogen (Fg) and von Willebrand factor (vWF) and are covalently linked to the bacterial cell wall via sortase A (SrtA). ${ }^{12-15}$ However, in view of intra-molecular folding of some MSCRAMMs, mostly having been studied in static conditions, many of these interactions may have different relevance in endovascular infections in the circulation. Indeed, we and others recently reported that $S$. aureus interactions via vWF are flow dependent which is in accordance with the well-known shear stress-controlled function of vWF. ${ }^{16,17}$

FnbpA and ClfA are two well-described surface adhesins of $S$. aureus. FnbpA is a Fn-binding protein with 11 Fnbinding repeats. ${ }^{18}$ Binding of FnbpA to Fn in static conditions is mediated by the $C D$ domain, the $A^{16+}$ domain and the hinge between the A domain and $B$ domain, which is capable of binding both Fn and Fg., ${ }^{3,5}$ Recent studies emphasize the significant contribution of FnbpA to bacterial adhesion and invasion of ECs by using Fn as a bridging molecule between the bacterium and the host endothelial Fn-receptor $\alpha 5 \beta 1$ integrin. $^{19-22}$ In static conditions, binding of FnbpA to ECs and their pro-inflammatory and pro-coagulant activation are primarily mediated by the $\mathrm{CD}$ domain. ${ }^{3}$ In vivo, it has been demonstrated that FnbpA-mediated binding to Fn is mainly crucial for S. aureus when invading ECs. ${ }^{19}$

ClfA is a staphylococcal surface protein with Fg-binding properties. The A domain of FnbpA shows sequence identity to the A domain of the Fg-binding protein ClfA and also exhibits Fg-binding activity. Both ClfA and FnbpA bind the $\gamma$-chain of $\mathrm{Fg}$ near the C-terminus ${ }^{6,8}$ and this interaction allows Fg to bridge $S$. aureus and ECs via the endothelial Fg-receptor $\alpha \mathrm{Vß3}$ integrin. ${ }^{23,24}$

It is, however, unclear how these interactions quantitatively translate in adhesive efficiency in flow conditions. Our group already described that adhesion of $S$. aureus to ECs and to sub-endothelial matrix in flow conditions is mediated via vWF and staphylococcal secreted vWF-binding protein (vWbp). ${ }^{17}$ Recently, we also found that a complex between vascular vWF, soluble vWbp and bacterial ClfA can be formed, which is responsible for $S$. aureus recruitment to inflamed (activated) endothelium. ${ }^{25}$

Lactococcus lactis are poorly pathogenic bacteria lacking adhesion molecules for human matrix proteins. However, L. lactis expresses its surface proteins in a similar way as S. aureus, using a LPXTG motif to anchor surface proteins to the peptidoglycan layer via SrtA. L. lactis expressing single staphylococcal surface proteins were reported before to study the contribution of single staphylococcal surface proteins in S. aureus IE. 3,5,24,26

In this study, we have investigated how ClfA, FnbpA and FnbpA sub-domains $\left(A, A^{16+}, A B C, C D\right)$ contribute to bacterial adhesion to various ligands present in injured vascular lesions. To validate the flow dependency of these interactions, we investigated the adhesion of L. lactis expressing ClfA, FnbpA or FnbpA sub-domains to Fn, Fg, intact and activated ECs and to fibrin. In view of the presence of Fg, Fn and a disintegrin and metalloproteinase with thrombospondin type 1 motif, member 13 (ADAMTS13) in plasma, our studies were performed both, in medium and in plasma, to account for these effects. Together, these experiments have enabled us to rank the importance of the major adhesive $S$. aureus receptors in bacterial recruitment in plasma to the inflamed and injured vessel wall and heart valve endothelium, central in IE.

\section{Materials and Methods}

\section{Bacterial Strains}

L. lactis strains ${ }^{24,26}$ were grown overnight at $37^{\circ} \mathrm{C}$ in M17 medium (Fluka, Sigma-Aldrich, Taufkirchen, Germany) supplemented with $0.5 \%$ glucose and $5 \mu \mathrm{g} / \mathrm{mL}$ erythromycin. Bacteria stocks were stored in M17 medium supplemented with $15 \%$ glycerol at $-80^{\circ} \mathrm{C}$. The strains used in this study are listed in - Supplementary Table S1, available in the online version. The original strain for all mutants was L. lactis subsp. cremonis 1363. The structural organization of FnbpA and its domains are presented in - Supplementary Fig. S1, available in the online version.

L. lactis strains have a documented membrane expression of ClfA and FnbpA, which is reported to be comparable to that of the corresponding adhesins in S. aureus Newman. ${ }^{24} \mathrm{~S}$. aureus strains were cultured at $37^{\circ} \mathrm{C}$ in tryptic soy broth (TSB) medium and stocks were stored in medium supplemented with $15 \%$ glycerol at $-80^{\circ} \mathrm{C}$.

\section{In Vitro Perfusion Experiments}

In vitro perfusion experiments were performed as described before. ${ }^{17,27}$ Glass coverslips $(24 \times 50 \mathrm{~mm}$, VWR International, Belgium) were coated with $100 \mu \mathrm{g} / \mathrm{mL}$ Fn (SigmaAldrich), $100 \mu \mathrm{g} / \mathrm{mL} \mathrm{Fg}^{21}$ (Sigma-Aldrich), $30 \mu \mathrm{g} / \mathrm{mL} \mathrm{rvWbp}^{27}$ or $50 \mu \mathrm{g} / \mathrm{mL}$ vWF A1-domain ${ }^{25}$ in a humidified container at room temperature for 4 hours. The coverslips were mounted in a micro-parallel plate flow chamber ${ }^{28}$ and perfused for 10 minutes with a high accuracy Harvard pump (PHD 2000 Infusion, Harvard Apparatus, Cambridge, Massachusetts, United States), generating flow rates of $500,1,000$ or $2,000 \mathrm{~s}^{-1}$. Bacteria were labelled with 5(6)-carboxy-fluorescein $\mathrm{N}$ hydroxysuccinimidyl ester (Sigma-Aldrich) (final concentration of $30 \mu \mathrm{g} / \mathrm{mL}$ for subsequent perfusion experiments) and diluted in Dulbecco's modified Eagle medium (DMEM) supplemented with $25 \mathrm{mM}$ 4-(2-hydroxyethyl)-1-piperazineethanesulfonic acid (HEPES) pH 7.5 and 10\% fetal bovine serum (FBS) to an $\mathrm{OD}_{600 \mathrm{~nm}}$ (optical density) of 0.65 or 1.2 corresponding to $5.0 \times 10^{7}$ or $10^{8}$ colony-forming unit (CFU)/ $\mathrm{mL}$, respectively. An approximate number of $7.0 \times 10^{7} \mathrm{CFUs}$ was used to assess bacterial adhesion to extracellular matrix proteins ( $\mathrm{Fg}$, fibrin, Fn), whereas $1.4 \times 10^{8} \mathrm{CFUs}$ were taken for the evaluation of adhesion events to EC layers.

Coated coverslips were perfused with labelled bacteria $\left(\mathrm{OD}_{600 \mathrm{~nm}} 0.65\right)$ with or without added rvWbp $(10-50 \mu \mathrm{g} / \mathrm{mL})$, 
$\mathrm{Ca}^{2+}(10 \mathrm{mM})$ and $\mathrm{Fg}(10-200 \mu \mathrm{g} / \mathrm{mL})$. Live images were recorded using an inverted fluorescence microscope (Axio-observer DI, Carl-Zeiss NV, Belgium) and read with a black and white camera (Carl-Zeiss Axio-Cam MRm). Images were digitally stored and fluorescence was measured with the Image J analysis software (National Institutes of Health, Bethesda, Maryland, United States) using a particle analysis module, both manually and by means of an elaborated macro. The intensity of fluorescence is reported as relative bacterial adhesion, expressed as a percentage of an internal control, in most case consisting of L. lactis-fnbpA adhesion, as indicated. In some cases, as mentioned, bacterial adherence was also defined by intensity measurement, as arbitrary fluorescence units of cumulative foci intensity, or by a surface coverage area analysis, referring to the area occupied by adhered bacteria.

\section{Bacterial Adhesion to Endothelial Cells}

Human umbilical vein endothelial cells (HUVECs) were isolated from fresh umbilical cords of healthy donors after taking informed consent, as described before. ${ }^{17,27}$ HUVECs were seeded on $1 \%$ gelatin (Sigma-Alderich)-coated plastic microscopic slides (Sarstedt, Nuembrecht, Germany) and grown to confluence. The slides were mounted in a microparallel plate flow chamber and ECs were perfused with fluorescently labelled bacteria in DMEM, containing $25 \mathrm{mM}$ HEPES pH 7.5 and 10\% FBS (supplemented DMEM) for 10 minutes at a shear rate of $1,000 \mathrm{~s}^{-1}$. When indicated, ECs were activated via perfusion with $10 \mu \mathrm{M} \mathrm{Ca}^{2+}$-ionophore A23187 (Sigma-Aldrich) for 5 minutes. Fluorescently labelled bacteria were suspended in supplemented DMEM $\left(10^{8} \mathrm{CFU} / \mathrm{mL}\right)$ or platelet-poor plasma (1:1 diluted in DMEM, containing $50 \mathrm{mM}$ HEPES pH 7.5 and 10\% FBS), with or without Fn $(100 \mu \mathrm{g} / \mathrm{mL})$, Fg $(20-200 \mu \mathrm{g} / \mathrm{mL})$, rvWbp (10$50 \mu \mathrm{g} / \mathrm{mL})$ and/or eptifibatide $(10 \mu \mathrm{g} / \mathrm{mL}$, GlaxoSmithKline, Brentford, UK) added to the perfusate. After perfusion of activated HUVECs with or without $30 \mu \mathrm{g} / \mathrm{mL}$ rvWbp in supplemented DMEM, ADAMTS13 string cleaving activity of vWF multimers was checked by post-perfusion with medium or plasma, as indicated. HUVECs were pre-perfused with rvWbp (activated ECs) or Fg (non-activated ECs), as indicated, to avoid strong secondary interactions shown in this study as agglutinated bacterial clumps, which in nonplasma environments complicated the adhesion process. Human plasma used in this study was constituted of pooled plasma from eight healthy donors. Before use, plasma was centrifuged at $1,000 \mathrm{rpm}$ at room temperature and was additionally filtered through $0.45 \mu \mathrm{m}$ sterile filter to avoid particles that would clog the perfusion chamber. Bacterial adhesion was recorded as described above.

\section{Bacterial Adhesion to Fibrin}

Glass coverslips $(24 \times 50 \mathrm{~mm}$, VWR International $)$ were coated with $50 \mu \mathrm{g} / \mathrm{mL}$ Horm collagen (Takeda, Linz, Austria) in a humidified container at room temperature for 4 hours, rinsed with HEPES buffer of $\mathrm{pH} 7.5$ and blocked with $5 \%$ bovine serum albumin dissolved in HEPES buffer of $\mathrm{pH} 7.5$ for 30 minutes. The coverslips were mounted in a micro-parallel plate flow chamber and perfused for 6 minutes with citrated whole blood at $1,000 \mathrm{~s}^{-1}$ to trigger platelet adhesion and primary platelet aggregation. After a wash step with HEPES buffer, $\mathrm{pH} 7.5$, the coverslips were perfused with re-calcified (16.4 $\mathrm{mM} \mathrm{CaCl} 2$, Sigma-Aldrich) plasma (plasma pool, 8 healthy donors) for 12 minutes at $150 \mathrm{~s}^{-1}$, to trigger fibrin formation on the adhered platelets. Then, after washing with the HEPES buffer, the fibrin layer was perfused with fluorescently labelled bacteria suspended in supplemented DMEM for 10 minutes at different shear rates (500, 1,000 and 2,000 s$\left.{ }^{-1}\right)$. Bacterial adhesion to the fibrin layer was recorded as described above.

\section{Static Agglutination Test}

Fluorescently labelled bacteria were diluted in DMEM or plasma to an $\mathrm{OD}_{600 \mathrm{~nm}}$ of 0.65 with or without $30 \mu \mathrm{g} / \mathrm{mL}$ rvWbp or Fg (50-200 $\mu \mathrm{g} / \mathrm{mL})$. Droplets of $500 \mu \mathrm{L}$ were placed on a glass coverslip and incubated for 5 minutes at room temperature. Live images were obtained using an inverted fluorescence microscope (Axio- observer DI, Carl-Zeiss NV, Belgium) and recorded with a black and white camera (CarlZeiss Axio-Cam MRm).

\section{Statistical Analysis}

All calculations were done with GraphPad Prism 7.0d (GraphPad Software, San Diego, California, United States). Groups were compared with the one-way analysis of variance or a two-tailed Student's $t$-test. All values are reported as mean \pm standard error of the mean. A $p$-value of $<0.05$ was considered significant $\left({ }^{*} p<0.05 ;{ }^{* *} p<0.01 ;{ }^{* * *} p<0.001 ;{ }^{* * * *} p<0.0001\right)$.

\section{Results}

\section{Adhesion of Lactococci Expressing FnbpA and ClfA to Fn, Fg and ECs in Flow}

The FnbpA sub-domains contribute to $S$. aureus adhesion to Fn and Fg in static conditions and, as shown before, the $C D$ domain of FnbpA mediates binding to Fn and the $A^{16+}$ domain binds both Fn and Fg. ${ }^{5}$ We first evaluated the influence of shear stress on the adhesion of L. lactis expressing FnbpA or ClfA to Fn and Fg in conditions where the native $L$. lactis-pIL253 control strain showed only minimal adhesion (-Fig. 1A, B). The expression of FnbpA in L. lactis allowed adhesion to $\mathrm{Fn}$ and $\mathrm{Fg}$ in flow, while ClfA selectively triggered adhesion to Fg ( $\mathbf{- F i g . 1 A}$, B), which was considerably higher than that of $L$. lactis-fnbpA (-Fig. 1B) $(p=0.0058)$. We further investigated in detail the role of staphylococcal FnbpA sub-domains for adhesion to Fn and Fg, taking $L$. lactis-fnbpA as an internal reference, set to $100 \%$, thus resulting in some quantitative differences (-Fig. 1A, B). In conclusion, the CD-domain bound selectively to Fn (compared to full length FnbpA, $p=0.039$ ) and ClfA to Fg. The $\mathrm{A}^{16+}$ domain bound both Fn and Fg. Or, the same domains controlling bacterial adhesion to Fn and Fg in static conditions also determine bacterial binding in flow. Of interest, L. lactis-clfA and $L$. lactis- $C D$ could serve as selective probes to measure bacterial binding to Fg and Fn, respectively. 


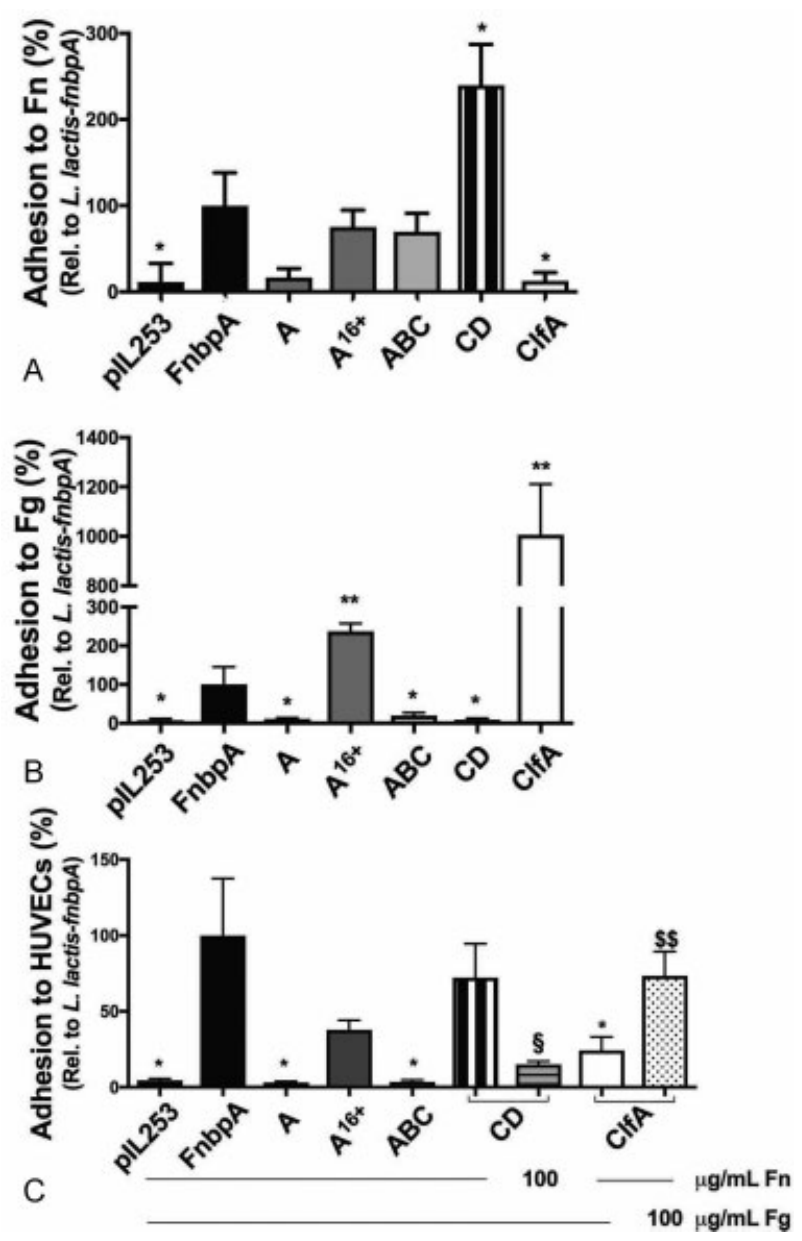

Fig. 1 L. lactis- $C D$ and L. lactis-fibronectin binding protein $A(f n b p A)$ bind primarily to fibronectin $(\mathrm{Fn})$ and L. lactis-clumping factor $A(c \mid f A)$ binds to fibrinogen $(\mathrm{Fg})$ under shear stress. $(\mathrm{A}-\mathrm{C})$ Micro-parallel plate flow chamber perfusion over coated $\mathrm{Fn}(\mathrm{A}, 100 \mu \mathrm{g} / \mathrm{mL})$ or $\mathrm{Fg}$ (B, $100 \mu \mathrm{g} / \mathrm{mL}$ ) and human umbilical vein endothelial cells (HUVECs) (C) with fluorescently labelled L. lactis-plL253, L. lactis-fnbpA, L. lactis-A, L. lactis-A $A^{16+}$, L. lactis- $A B C$, L. lactis-CD and L. lactis-ClfA strains at a shear rate of $1,000 \mathrm{~s}^{-1}(n \geq 6)$. Where indicated, $\mathrm{Fn}$ or Fg were added to the perfusate. All results are expressed as mean \pm standard error of the mean (SEM). ${ }^{*} p<0.05,{ }^{* *} p<0.01$ compared to L. lactis-fnbpA, $\S p<0.05$ compared to L. lactis-CD, $\$ \$ p<0.01$ compared to L. lactis-clfA.

Therefore, L. lactis expressing ClfA, FnbpA and its subdomains were selected to study these adhesins in bacterial recruitment to intact ECs in flow conditions. L. lactis-fnbpA bound to ECs via the $C D$ domain and the $A^{16+}$ domain (-Fig. 1C). Because of the strong binding via the $C D$ domain, we suspected that the adhesion observed for L. lactis-fnbpA was mediated via EC-bound Fn. Indeed, perfusions with an excess of Fn, added to the perfusate to saturate the L. lactis$C D$ Fn integrin receptor(s) almost abrogated adhesion (-Fig. 1C). In contrast, L. lactis-clfA showed little interaction with ECs (-Fig. 1C), but adding Fg to the perfusate restored the ClfA-mediated adhesion to comparable levels as measured for L. lactis-fnbpA, taken as an internal control set at $100 \%$ recruitment (-Fig. 1C). Moreover, there was no difference if $\mathrm{Fg}$ was added to the perfusate or pre-perfused over ECs, showing that Fg pre-perfusion is also a valid strategy
(-Supplementary Fig. S3A, available in the online version). Inclusion of the integrin receptor antagonist integrilin in the perfusate confirmed complete inhibition of L. lactis-clfA binding to ECs (not shown). We conclude, that ClfA selectively mediates bacterial adhesion to ECs via EC-bound Fg.

\section{Interaction of $L$. Lactis-fnbpA and L. Lactis-clfA with Fg versus Fibrin in Flow}

S. aureus is known to adhere to platelet-fibrin clots, present in endocarditis lesions. ${ }^{29,30}$ Therefore, the interactions of $L$. lactis-clfA with Fg and fibrin were presently compared in flow, information currently lacking for fibrin. Adhesion of $L$. lactis-clfA to coated $\mathrm{Fg}$ was homogenous, bacteria being equally distributed and it was shear stress-sensitive, gradually increasing with shear rates rising from 500 to $2,000 \mathrm{~s}^{-1}$ ( - Fig. 2A, $p=0.045$ ). In addition, adhesion to Fg was highly selectively mediated by ClfA ( - Fig. 2A, left panel), in agreement with findings shown in - Fig. 1 .

The adhesion to fibrin occurred to the preformed fibrin fibres and clots and resulted in massive L. lactis-clfA adhesion after 10 minutes (-Fig. 2B, right panel). The binding of $L$. lactis-clfA to fibrin went up when the shear rate rose from 500 to $1,000 \mathrm{~s}^{-1}$ ( $\left.p=0.0024\right)$, but dropped at $2,000 \mathrm{~s}^{-1}$ to the same degree as observed for the binding of L. lactis-fnbpA and L. lactis-CD ( - Fig. 2B, left panel). Overall, L. lactis-clfA interacted with coated $\mathrm{Fg}$ and fibrin remarkably differently. Adhesion to fibrin was more focal and resisted the highest shear rate tested less well ( $\mathbf{- F i g . ~ 2 B}$ ), hence appeared to be not more selective than the binding of L. lactis-fnbpA and $L$. lactis- $C D$ to fibrin. It may reflect a situation in which these fibrin higher-ordered structures form a kind of three-dimensional scaffold that does not experience high shear forces at the site of lesions.

\section{ClfA Binds to Fibrin via the Fg $\mathbf{y}$-Chain}

The Fg $\gamma$-chain has been identified as the interaction site for ClfA. ${ }^{8}$ In view of the different interaction profile of ClfA with Fg and fibrin, we have tested whether blocking the Fg $\gamma$-chain with $\mathrm{Ca}^{2+}$ would affect fibrin-L. lactis-clfA interactions. $\mathrm{CaCl}_{2}$ (10 mM) inhibited comparably L. lactis-clfA adhesion to Fg and fibrin (-Fig. 3), illustrating the involvement of the Fg $\gamma$-chain in both cases, further documenting the availability of this binding site in fibrin clots. We previously showed that ClfA interacts with rvWbp to promote vWF-mediated adhesion to ECs and sub-endothelial matrix. Interestingly, rvWbp, however, did not inhibit the binding of L. lactis-clfA to Fg or fibrin (-Fig. 3). In contrast, L. lactis-clfA binding to Fg was even enhanced by adding 10 to $30 \mu \mathrm{g} / \mathrm{mL}$ rvWbp (- Fig 3A). These findings are compatible with the formation of a ternary complex between $\mathrm{Fg}$, vWbp and ClfA, where vWbp stabilizes the binding between Fg and ClfA (-Figs. 3A and $\mathbf{4}$ ), in further agreement with its known binding to $\mathrm{Fg}$, when it is part of the staphylothrombin complex, capable of Fg conversion. ${ }^{31}$ Such a complex seems to play a lesser role in L. lactis-clfA adhesion to fibrin ( - Fig. 3B). Finally, saturation of L. lactis-clfA with Fg $(100 \mu \mathrm{g} / \mathrm{mL})$ interferes with its binding to fibrin (-Fig. 3B), illustrating that an excess of soluble Fg competes with fibrin for binding to bacterial ClfA. We conclude that ClfA binds to fibrin 


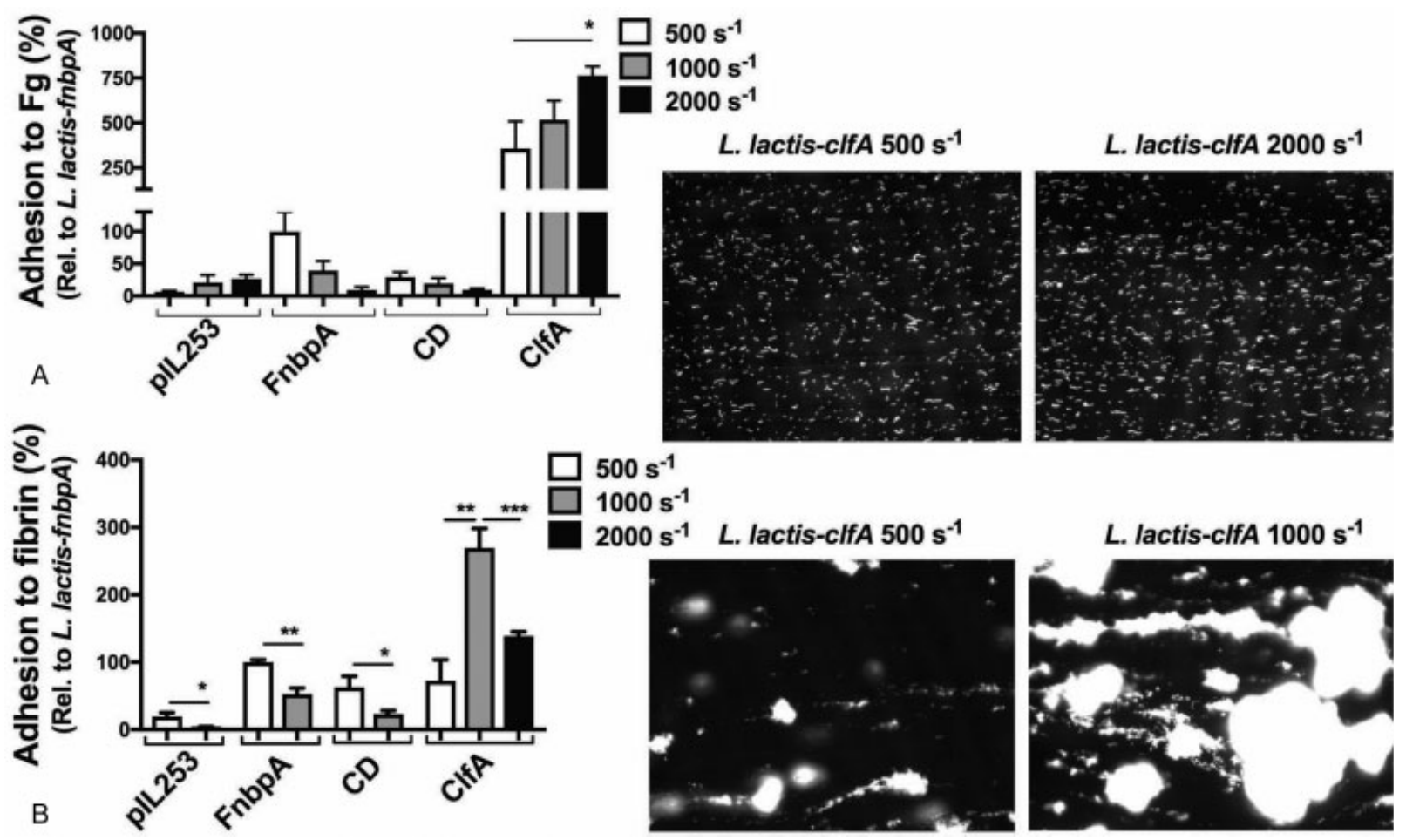

Fig. 2 L. lactis expressing clumping factor $A(C l f A)$ binds to fibrinogen $(\mathrm{Fg})$ and fibrin under shear stress. Micro-parallel plate flow chamber perfusion over coated $\mathrm{Fg}(\mathrm{A}, 100 \mu \mathrm{g} / \mathrm{mL})$ and fibrin (B) with fluorescently labelled L. lactis-plL253, L. lactis-fnbpA, L. lactis-CD and L. lactis-clfA strains at a shear rate of $500,1,000$ or $2,000 \mathrm{~s}^{-1}$. Representative images show L. lactis-clfA adhering to $\mathrm{Fg}(n>6)$ and fibrin $(n>4)$ at shear rates of 500 and $2,000 \mathrm{~s}^{-1}$. All results are expressed as mean \pm standard error of the mean (SEM). ${ }^{*} p<0.05,{ }^{* *} p<0.01,{ }^{* * *} p<0.001$.

via the Fg $\gamma$-chain, avidly enough to mediate bacterial adhesion to fibrin-rich clots or lesions. Additionally, no enhancement of adhesion to fibrin in the presence of vWbp emphasizes no further requirement for the staphylothrombin complex formation towards more Fg conversion.

\section{The Effect of Fg on vWbp-CIfA Interactions}

We previously reported that adhesion of S. aureus to ECs and to the sub-endothelial matrix under shear stress occurred through the A1 domain of vWF, interacting with vWbp. ${ }^{25,27}$ Interactions exist between vWbp, Fg and ClfA (-Figs. 3A and 4 ) and $S$. aureus binding to $\mathrm{EC}$ results from complex formation between vascular vWF, soluble vWbp and bacterial ClfA, ${ }^{25}$ recruiting $S$. aureus to inflamed (activated) endothelium. We have, therefore, further investigated how Fg would affect the interaction between vWbp and ClfA. ECs were activated by a $\mathrm{Ca}^{2+}$-ionophore to stimulate release of vWF, which was then retained on the EC surface, in the absence of ADAMTS13.

In agreement with earlier findings, ${ }^{25}$ soluble vWbp $(30 \mu \mathrm{g} / \mathrm{mL})$ increased adhesion of $L$. lactis-clfA to the $\mathrm{A} 1$ domain of $\mathrm{vWF}$. Adhesion was further enhanced by adding Fg up to $100 \mu \mathrm{g} / \mathrm{mL}$ (-Fig 5A). The - Supplementary Fig. $\mathbf{S 2}$ (available in the online version) illustrates how 30 to $200 \mu \mathrm{g} / \mathrm{mL}$ Fg causes L. lactis-clfA agglutination in a buffer environment. Hence, rapid formation of these larger, more stable bacterial clumps has a positive effect on the bacterial adhesion ( $\mathbf{- F i g . ~ 5 A}$, left and right panels). This observation explains why Fg further raised adhesion of $L$. lactis-clfA to the A1 domain of vWF, i.e. in the absence of EC receptors for Fg.
Whereas we observed that L. lactis-clfA firmly bound to coated vWbp in a homogenous pattern ( - Fig. 5B, right panel), the Fg-mediated agglutination of L. lactis-clfA did not further enhance bacterial interactions with coated vWbp, even when we still observed the formation and adhesion of agglutinated bacterial clumps (- Fig. 5B). However, the adhered clumps were unstable, reflecting weaker interactions between Fg, ClfA and rvWbp, when the latter was coated and less accessible for stabilizing interactions between ClfA and Fg. This was not further investigated. Therefore, these data indicate that vWbp-ClfA and Fg-ClfA are not mutually exclusive, but that the final outcome of vWbp-Fg-ClfA interactions for bacterial recruitments depends on the degree of bacterial agglutination. - Supplementary Fig. S2 (available in the online version) describes the agglutination issue in larger detail.

\section{How do Fg and vWbp Mediate Adhesion of L. Lactis-clfA to Activated Endothelial Cells}

Next, we studied how ClfA would mediate bacterial adhesion to activated ECs by simultaneously focusing on the vWF/ vWbp and the integrin receptor-Fg pathways.

In the absence of vWbp or Fg, L. lactis-clfA weakly adhered to activated ECs and hardly more than the adhesion of the negative control pIL253 (-Fig. 6A). However, addition of either low vWbp or low Fg concentrations to the perfusate increased adhesion in both cases to comparable levels, illustrating equivalence for $\mathrm{vWF} / \mathrm{vWbp}$ and integrin receptor-Fg mediated binding, analysed separately. The integrin inhibitor integrilin abrogated the Fg-mediated L. lactis-clfA 

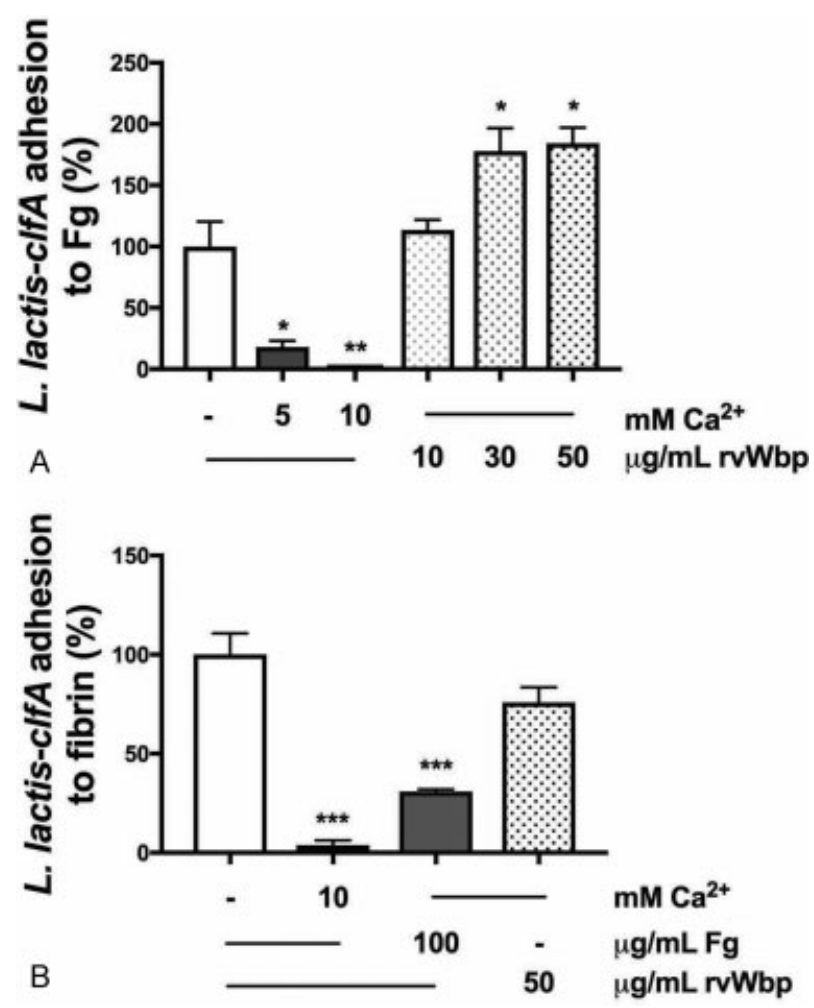

Fig. 3 L. lactis expressing clumping factor A (ClfA) binds to fibrin under shear stress via the $y$-chain of fibrinogen (Fg). Micro-parallel plate flow chamber perfusion over coated $\mathrm{Fg}(\mathrm{A}, 100 \mu \mathrm{g} / \mathrm{mL}, n>6)$ and fibrin $(B, n>4)$ with fluorescently labelled $L$. lactis-clfA strain at a shear rate of $1,000 \mathrm{~s}^{-1}$. Where indicated, $\mathrm{Ca}^{2+}$, Fg or von Willebrand factor-binding protein (rvWbp) were added to the perfusate. All results are expressed as mean \pm standard error of the mean (SEM). ${ }^{*} p<0.05,{ }^{* *} p<0.01$,

${ }^{* * *} p<0.001$ compared to L. lactis-clfA (negative control).

adhesion. In other words, the binding of L. lactis-clfA to activated ECs via $\mathrm{vWF} / \mathrm{vWbp}$ and integrin receptor-Fg is comparable and is furthermore similar to the recruitment of $L$. lactis-fnbpA, used as an internal reference being set to $100 \%$. Taking into account the agglutination of L. lactis-clfA by Fg (-Supplementary Fig. S2, available in the online version), the binding of $L$. lactis-clfA via $\mathrm{vWF} / \mathrm{vWbp}$ occurred via single bacteria and that to EC-bound Fg via agglutinated clumps (-Fig. 6A, right panel).

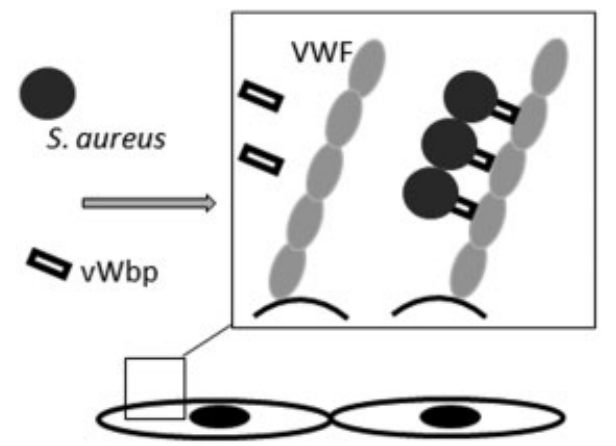

In the more complex condition where vWbp and Fg were simultaneously added to the perfusate, we observed that $L$. lactis-clfA adhesion to activated ECs was never higher than that of the internal control, i.e. combining pathways did not lift interaction efficiencies over those found for the single pathways. Both, when rvWbp was pre-perfused or added to the perfusate, the degree of interaction was similar and only weakly but positively influenced by the additional presence of Fg (-Fig. 6B). At higher degrees of agglutination (see -Supplementary Fig. S2, available in the online version), the combined presence of rvWbp and Fg resulted even in the inhibition of bacterial recruitment. Or, in this complex environment, dependent on multiple interactions between vWF, rvWbp, Fg and ClfA, no additive effects were found for the vWF/vWbp and the EC-bound Fg pathways.

\section{The Adhesion of L. Lactis-clfA to Endothelial Cells in Plasma}

In the previous figures, L. lactis-clfA interactions with ECs were largely controlled by Fg-induced agglutination of L. lactis-clfA. However, in plasma where Fg concentrations are considerably higher and other plasma proteins can participate in the agglutination process, we did not observe agglutination any longer over the time interval of our incubations (-Supplementary Fig. S2, available in the online version). In addition, plasma contains the metalloproteinase ADAMTS13. Because we had already shown that ADAMTS13 decreases the vWF-mediated $S$. aureus adhesion to ECs by $60 \%$, as a result of vWF multimeric string cleavage by ADAMTS13, ${ }^{27}$ it became necessary to assess the adhesion of $L$. lactis-clfA to ECs while introducing flow and plasma as two additional factors.

Perfusions during 10 minutes over ECs in 50\% plasma show that ionophore-activation of ECs reduced the degree of $L$. lactis-fnbpA adhesion by about 50\% (-Fig. 7A). At the same time, the adhesion of $L$. lactis-clfA is considerably higher in plasma and is hardly affected by EC-ionophore activation, in agreement with the interpretation that Fg-binding to ECs is independent of EC-ionophore treatment. Reperfusions of activated ECs with rvWbp or inclusion of rvWbp in the perfusate further enhanced the adhesion, assessed after 10 minutes of perfusion time. To express bacterial adherence more quantitatively, we compared bacterial adherence as 'surface coverage' after perfusions of 10 minutes in the absence and presence

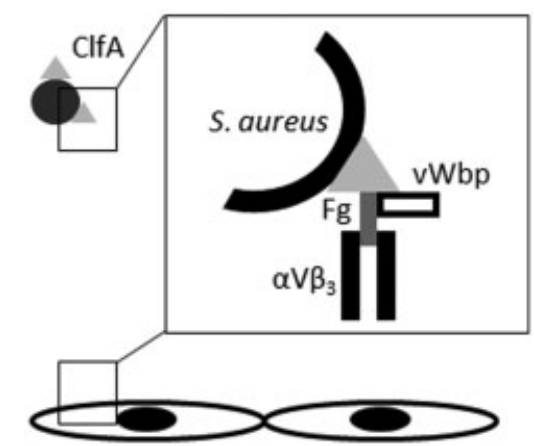

Fig. 4 Schematic model indicating how binding of clumping factor $A$ (ClfA) promotes bacterial attachment to endothelial cells (ECs) under shear stress, ClfA binds via von Willebrand factor (vWF)-binding protein (vWbp) to vWF present on activated ECs. Via a second pathway, ClfA binds to the integrins, mediated by fibrinogen $(\mathrm{Fg})$. vWbp is part of a complex formed with $\mathrm{Fg}$ and ClfA. 


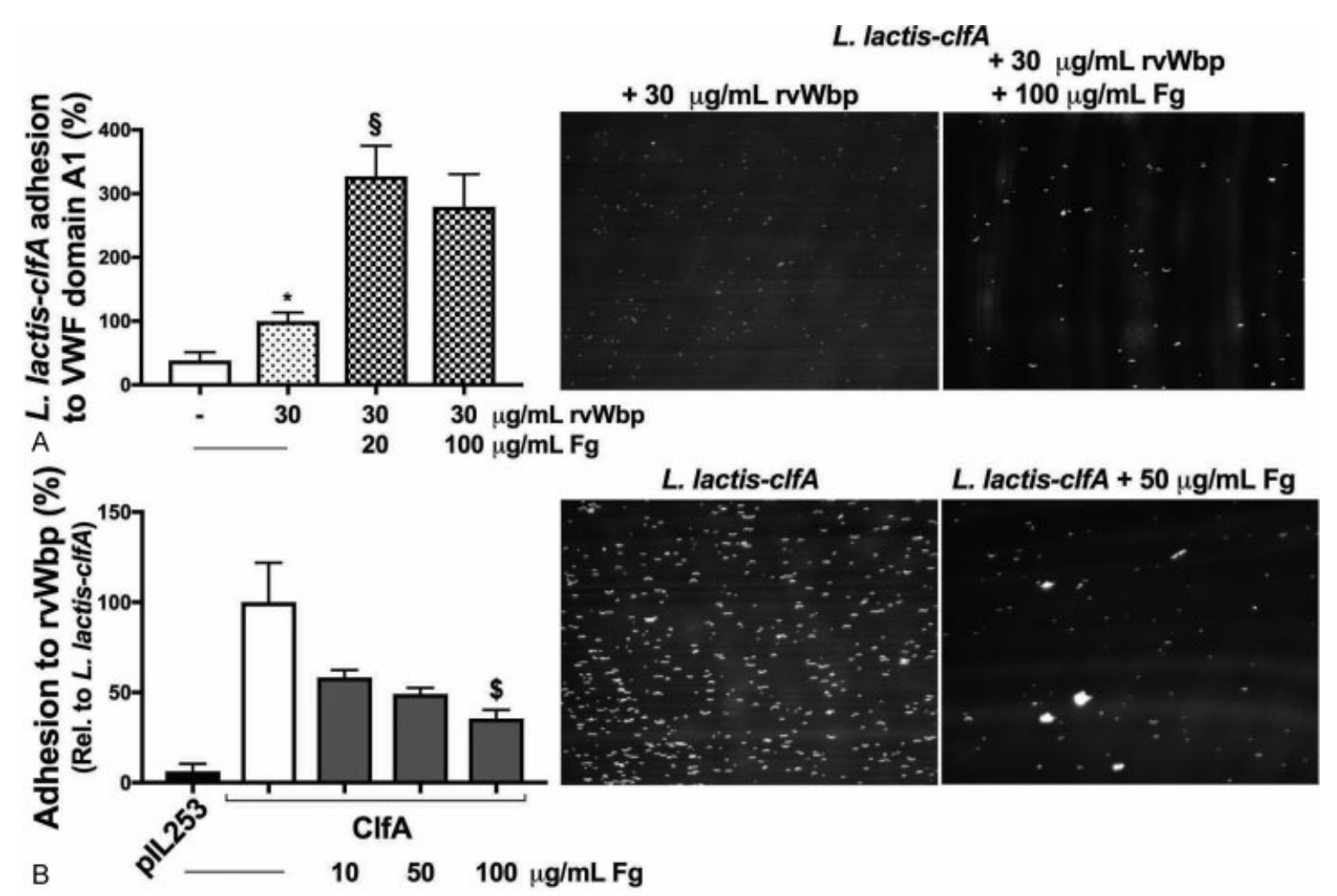

Fig. 5 Aggregate formation of L. lactis expressing clumping factor A (ClfA) with fibrinogen ( $\mathrm{Fg}$ ) enhances adhesion to von Willebrand factor (vWF) A1-domain in the presence of vWF-binding protein (rvWbp), but decreases adhesion to coated rvWbp. Micro-parallel plate flow chamber perfusion over coated vWF A1-domain (A, $50 \mu \mathrm{g} / \mathrm{mL}, n>4$ ) and over rvWbp (B, $50 \mu \mathrm{g} / \mathrm{mL}, n>6$ ) with fluorescently labelled L. lactis-p/L253 and/ or L. lactis-clfA strains at a shear rate of $1,000 \mathrm{~s}^{-1}$. Where indicated, rvWbp and/or $\mathrm{Fg}$ were added to the perfusate. Representative images of $L$. lactis-clfA adhering to VWF A1-domain (A) and to rVWbp (B). All results are expressed as mean \pm standard error of the mean (SEM). ${ }^{*} p<0.05$ compared to L. lactis-clfA, ${ }^{*} \$ p<0.05$ compared to L. lactis-clfA $+30 \mu \mathrm{g} / \mathrm{mL}$ rvWbp, $\$ p<0.05$ compared to L. lactis-clfA.

of plasma. Hence, - Fig. 7A confirms that the potency of L. lactis-fnbpA adhesion strongly decreases in plasma, when assessed on ionophore-activated ECs. In contrast, L. lactis-clfA adhesion was as strongly stimulated by plasma as it was by Fg in the absence of further plasma proteins. Because, depending on the individual ECs chosen, the residual contribution of vWbp to bacterial adhesion in plasma appeared to be variable, we have assessed the impact of the vWF pathway in plasma more precisely, as shown in -Fig. 7B. To that end, we first perfused L. lactis-clfA over activated ECs and then added a wash-in step with medium or with plasma. We observed no difference in the degree and kinetics of adhesion when L. lactisclfA was perfused and washed-in with medium or plasma (-Fig. 7B, left panel). However, when vWbp was supplemented to the medium, an expected increased adhesion of $L$. lactisclfA was observed after the wash-in step. The wash-in step in plasma, where ADAMTS13 was able to cleave the EC-bound vWF strings, progressively decreased L. lactis-clfA adhesion to the activated ECs in the presence of vWbp to about $30 \%$ of the signal in the absence of plasma (-Fig. 7B, left panel).

When plasma was mixed with L. lactis-clfA and perfused over activated ECs for different time intervals before the wash-out, these different time points clearly revealed an increased adhesion when vWbp was present, but also illustrated that the cumulative adhesion during the first 4 minutes was reversed between 4 and 8 minutes (- Fig. 7B, right panel), in agreement with ADAMTS13-mediated vWF cleavage in this time frame. These experiments illustrate that the standard perfusion time of 10 minutes are long enough to enable ADAMTS13-mediated vWF-cleavage, largely reversing the vWF-dependent adhesion.

Or, although vWbp addition evidenced the residual bacterial binding via the $\mathrm{vWF} / \mathrm{vWbp}$ pathway (-Fig. 7B), i.e. to the vWF A1 domain still present on ECs after ADAMTS13 cleavage, this residue is small compared to the binding via the EC-bound Fg-ClfA pathway. This explains why the 'residue binding' may go unnoticed in some cases if compared to the integrin-Fg-ClfA pathway.

\section{Discussion}

To initiate IE, blood-borne $S$. aureus needs to colonize the endothelium via adhesion to the heart valve surface, itself subject to turbid blood flow. Both S. aureus ClfA and FnbpA are reported to be central players in bacterial adhesion to the endothelium. 4,5,11,20,32 We have, therefore, presently evaluated the adhesive properties for these both adhesins in vitro, in conditions more closely mimicking the contact interface between bacteria and the heart valve endothelium. To this end, perfusion studies with bacteria were performed over layers of confluent ECs, exposing them to variable mild shear forces in a plasma matrix. Bacteria consisted of several 


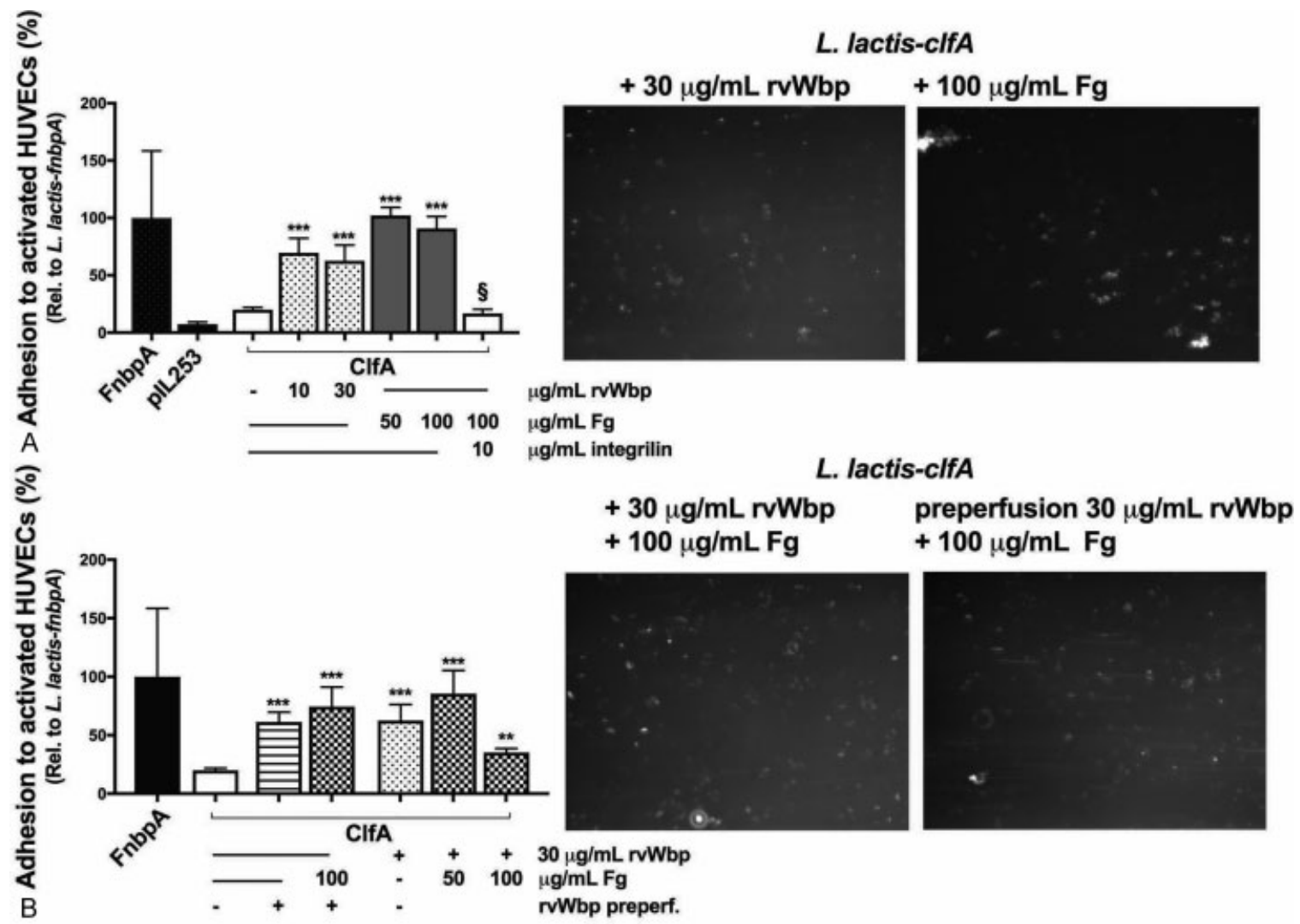

Fig. 6 Both fibrinogen $(\mathrm{Fg})$ and von Willebrand factor-binding protein (vWbp) mediate the adhesion of L. lactis expressing clumping factor $A$ (ClfA) to activated endothelial cells (ECS) in flow conditions. (A, B) Micro-parallel plate flow chamber perfusions of L. lactis-pIL253, L. lactis-clfA and L. lactis-fnbpA strains over ECs activated by a 5-minute perfusion with $\mathrm{Ca}^{2+}$-ionophore $(10 \mu \mathrm{M})$ at a shear rate of $1,000 \mathrm{~s}^{-1}$. Where indicated, rvWbp, Fg and/or integrilin were added to the perfusate $(n>6)$. (A, B) Representative images of $L$. lactis-clfA perfusion in the presence of rvWbp or Fg. All results are expressed as mean \pm standard error of the mean (SEM). ${ }^{* *} p<0.01$ and ${ }^{* * *} p<0.001$ compared to L. lactis-clfA, $\S p<0.001$ compared to L. lactis-clfA $+100 \mu \mathrm{g} / \mathrm{mL} \mathrm{Fg}$.

L. lactis strains, individually expressing ClfA or FnbpA (and its domains) to a level comparable to that found in $S$. aureus Newman. ${ }^{24}$ In the absence of plasma, these studies revealed that ClfA mediates bacterial recruitment to endothelium via vWF/vWbp and via EC-bound Fg with comparable efficiency, furthermore similar to the efficiency for the FnbpA/Fn pathway. This balance, however, is strongly affected by the high $\mathrm{Fg}$ concentration and by the presence of ADAMTS13 in plasma, defining ClfA as the most relevant adhesin in plasma. These findings can explain why ClfA, but not FnbpA, is a potent initiator of IE in animal models. ${ }^{19,24}$

ClfA binds Fg via its A domain, which recognizes the $\gamma$-chain of $\mathrm{Fg}^{8}$ and this interaction allows $\mathrm{Fg}$ to bridge S. aureus ClfA and the platelet receptor $\alpha \mathrm{IIb} \beta 3 .^{33} \mathrm{ClfA}$ also assures fibrin monomer binding to this receptor, ${ }^{34}$ facilitating S. aureus-triggered platelet aggregation. We have presently found that ClfA binding to fibrin, indeed, also occurs via its $\gamma$-chain, which is in agreement with the existence of circulating complexes between S. aureus, platelets and fibrin strands. ${ }^{30}$ Recently, it was shown that $\mathrm{Fg}$ also supports $S$. aureus adhesion to ECs via cross-linking ClfA to the endothelial integrin $\alpha \mathrm{VB3}$ integrin $^{23,24}$ a function partially overlapping that of staphylococcal FnbpA. FnbpA binds both $\mathrm{Fn}$ and $\mathrm{Fg}$, findings confirmed in this study and explained by the bridging of bacterial FnbpAvia Fn to the endothelial Fn receptor integrin $\alpha 5 ß 10^{3,24}$
Yet, even when these findings in perfused medium comply with previous static in vitro experiments of bacterial binding to ECs, showing predominant binding via the FnbpA receptor and only to a minor extent via ClfA, ${ }^{32}$ such data conflict with the more predominant role for ClfA in vivo. ${ }^{19} \mathrm{IE}$ models in the rat underscored a higher propensity for ClfA over FnbpA in heart valve colonization. ${ }^{19,26}$ In counterpart though, FnbpA triggered cell internalization in vitro and in vivo, leading to bacterial persistence in ECs, vegetations and peripheral organs. ${ }^{19}$ This interpretation is in further agreement with Kerrigan et al who described that, even when FnbpA and ClfA both bind Fg in static conditions, ClfA but not FnbpA activates and aggregates platelets via $\mathrm{Fg}$ bridging under shear stress. ${ }^{33}$ To further validate the significance of our adhesion data for ClfA, we have compared the degree of adhesion for L. lactis-clfA with that of wild-type $S$. aureus, making use of a ClfA deletion mutant (-Supplementary Fig. S3B, available in the online version). Thus, it allowed us to judge on the relative contribution of ClfA-Fg interactions to the adhesion of both strains of bacteria. This approach confirmed that the separate measurement of these interactions in the context of L. lactis was representative for their relevance in S. aureus. All interactions in this study were analysed in flow, at various shear rates and formal comparisons were made for the efficiencies 

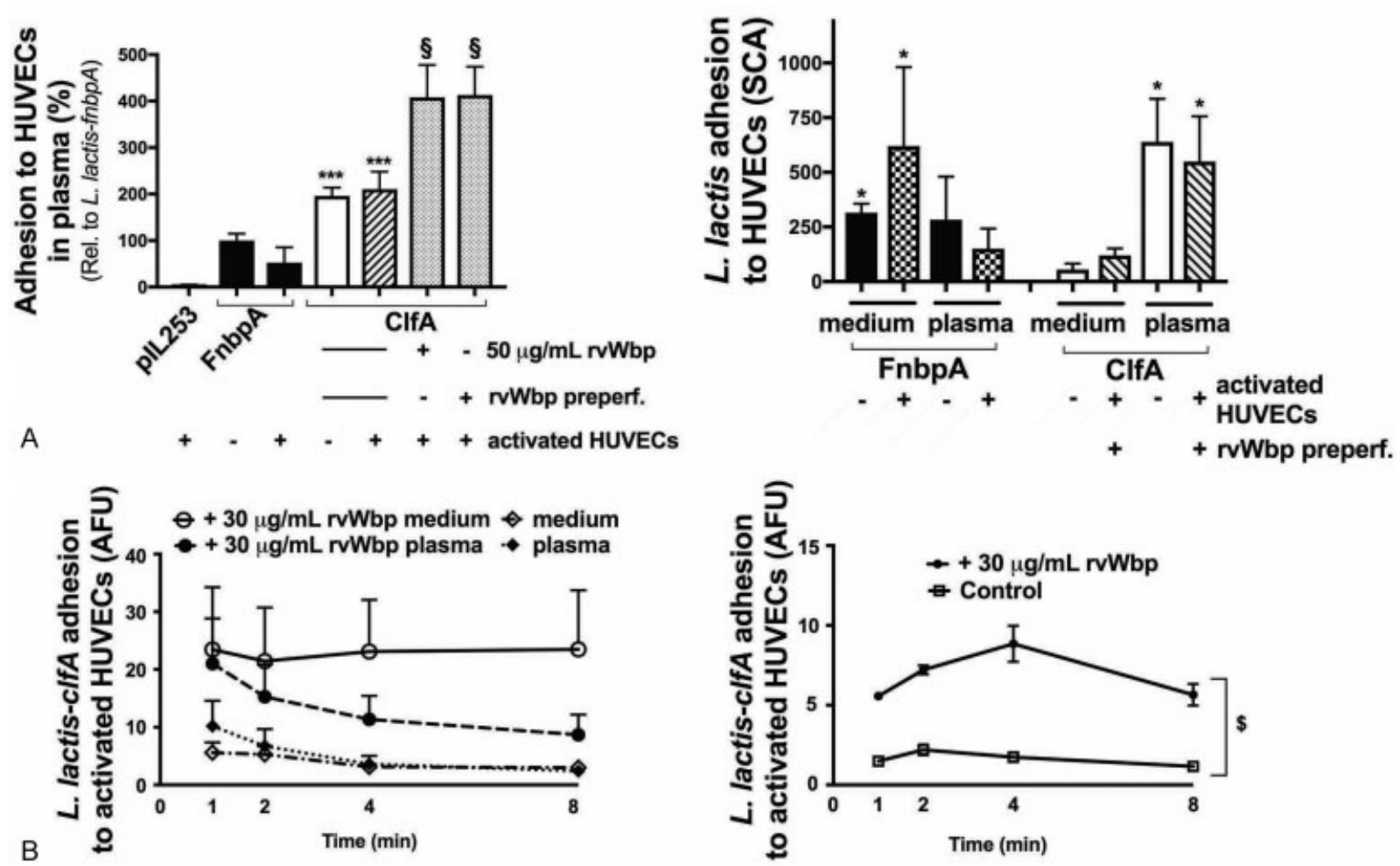

Fig. 7 Adhesion of L. lactis expressing fibronectin binding protein A (FnbpA) and clumping factor A (ClfA) to endothelial cells in plasma and medium conditions. (A) Micro-parallel plate flow chamber perfusions of fluorescently labelled L. lactis-pIL253, L. lactis-fnbpA and L. lactis-clfA strains over endothelial cells (ECs) in plasma (A, left panel) and medium compared to plasma (A, right panel, $n>4$, represented as surface coverage area $[S C A])$ at a shear rate of $1,000 \mathrm{~s}^{-1}$. Where indicated (A, B), ECs were activated with Ca ${ }^{2+}$-ionophore $(10 \mu \mathrm{M})$, and rvWbp (30 or $50 \mu \mathrm{g} / \mathrm{mL}$ ) was added to the perfusate or pre-perfused. (B) L. lactis-clfA perfusion over activated ECs followed by a perfusion of medium or plasma. Adhesion evaluated at different time points $(n=4$, represented as arbitrary fluorescent units $[\mathrm{AFU} / \mathrm{x} 1000])$. All results are expressed as mean \pm standard error of the mean (SEM). ${ }^{* * *} p<0.001$ compared to L. lactis-fnbpA (resting cells, normalization control), $\S p<0.05$ compared to L. lactis-clfA (activated ECs), ${ }^{*} p<0.05$ compared to L. lactis-clfA in medium (resting ECs) and $\$ p<0.01$.

of ClfA and FnbpA in bacterial adhesiveness to endothelium, by selecting $L$. lactis as the bacterial carrier for each adhesin individually. However, our findings showed that most domain interactions, presently studied during L. lactis recruitment to ECs were hardly flow-controlled, i.e. occurred with relative affinities, similar to those previously measured in static conditions. ${ }^{5,24}$ This suggests that the apparent conflicting roles of FnbpA and ClfA in vitro and in vivo ${ }^{19,33}$ are shear-independent and rather reflect the different environment in which such studies are performed, i.e. in buffer/ medium versus animal models. We have, therefore, also performed our studies in the presence of plasma and plasma proteins to study flow-controlled bacterial recruitment.

Nonetheless, to our surprise, we found that shear stress increases the interaction between ClfA and insolubilized Fg and to some degree also between ClfA and fibrin. These findings are suggestive of multi-domain interactions but also hint to a flow-dependent role of ClfA in the early phase of S. aureus infection, during its recruitment to EC integrinbound Fg. The existence of inter-independent binding sites on ClfA for Fg and for vWbp further documented the capacity of ClfA to participate in multi-domain interactions. This was further illustrated in this study by the enhanced recruitment of L. lactis-clfA onto coated VWF A1 domain, where Fg enhanced the vWbp-mediated binding of this strain, as also schematically represented in $\boldsymbol{- F i g . ~} \mathbf{4}$. Nevertheless, our findings made clear that plasma components rather than shear forces per se were the prime determinant of the adhesion efficiency.

Recently, we showed that $S$. aureus adhesion to vWF was shear-dependent and mediated by staphylococcal vWbp, an interaction equally dependent on S. aureus ClfA. ${ }^{17} \mathrm{vWbp}$ is not only an adhesive molecule but also acts as a coagulation-inducing bacterial protein, which is capable of forming a complex with human pro-thrombin, a property it shares with staphylocoagulase (Coa). ${ }^{35,36}$ Both secreted staphylococcal coagulases thus form a pro-thrombinase complex directly converting soluble $\mathrm{Fg}$ into fibrin clots. ${ }^{37,38}$ As mentioned above, bridging platelet $\alpha \operatorname{IIb} \beta 3$ and bacterial ClfA via fibrin monomers contributes to platelet activation and aggregate formation in a forming vegetation, ${ }^{34}$ but the present work also explicits how fibrin directly contributes to $S$. aureus recruitment in that process. The more important ClfA-mediated adhesion to fibrin at low shear rates is in agreement with histological observations which demonstrated fibrin deposition in the valvular sinus of non-infected heart valve prosthesis, a site of low shear stress. ${ }^{39}$ 
Taking into consideration that ClfA not only mediates interactions with $\mathrm{Fg}$ and fibrin, but via vWbp also with vWF, we have performed additional perfusion studies in plasma, to rationalize contributions by the various ligandreceptor couples, yet omitting platelets, the impact of which we had demonstrated before in flow, ${ }^{25}$ also to keep our models apprehensive. In these studies, we have used the non-selective but Fn-dependent adhesion of L. lactis-fnbpA as an internal reference to incorporate both plasma-Fn and plasma-Fg effects. A major functional characteristic of ClfA is its propensity to cause bacterial agglutination, a property strongly determining the degree of bacterial adhesiveness in various flow studies. Such was also the case in our studies, but in plasma, at least over fairly short time intervals, bacterial agglutination did not occur, allowing us to minimize disturbances by agglutination, such as observed in perfusions lacking plasma. Combined, the various perfusions performed over coated $\mathrm{Fg}$ and (ionophore-activated) EC layers revealed that the role of FnbpA was several-fold reduced in plasma compared to that of ClfA in triggering bacterial adhesion. Moreover, during sepsis, inflamed endothelium will expose temporarily retained vWF multimers. We had shown before that ADAMTS13 rapidly degrades these multimers, thus reducing bacterial adhesion by $60 \% .{ }^{27}$ We presently found that even in plasma, the ClfAmediated bacterial adhesion to residual vWF can still be higher than that of the binding measured in the absence of vWbp. Yet, ADAMTS13 rapidly reduces the importance of vWF-vWbp-ClfA interactions, thus favouring the EC-bound Fg-ClfA pathway. Hence, in plasma, where Fg saturates both FnbpA and ClfA and where ADAMTS13 quickly degrades vWF, the Fg-ClfA interaction is as performant as it is in the absence of plasma, when the perfusate is supplemented with Fg.

Clinical studies reveal that vWF is increased, leading to consumption of ADAMTS13 in sepsis patients. ${ }^{40,41}$ As a result, in sepsis and IE, the vWF/ADAMTS13 ratio rises, which may yet favour the importance of the $\mathrm{vWF} / \mathrm{vWbp}$ pathway during S. aureus adhesion to valvular and peripheral ECs. ${ }^{16,27}$ This remains to be further documented, but ClfA has been shown to be critical for valve colonization in rats with experimental IE. ${ }^{19,24}$ Blocking the Fg binding site of FnbpA did not alter Fn binding and cell internalization in vitro, whereas valve colonization in vivo was abrogated. ${ }^{19}$ Collectively, these findings show that binding to Fg and Fn are both required for colonization and invasion of heart valves, respectively. ${ }^{19}$ Therefore, even when our results reveal a smaller capacity for $S$. aureus to adhere to ECs in plasma via FnbpA, it seems important to consider that the propensity of infection is highly sufficient in vivo, the Fn-mediated binding of bacteria not being negligible.

In conclusion, our work has confirmed that both FnbpA and ClfA can contribute to S. aureus adhesion to ECs and fibrin in flow conditions. However, in plasma, the importance of FnbpA is reduced more strongly than that of ClfA by the high plasma Fg concentrations. Despite of vWF breakdown on inflamed endothelium by ADAMTS13 and high Fg concentrations in plasma, ClfA can mediate significant bacterial adhesion via both the vWF/vWbp and EC-integrin/Fg pathways. Our data suggest that novel therapeutic pharmacological or vaccination strategies should address these bacterial pathways specifically in order to reduce the pathogenicity of $S$. aureus.

\section{What is known about this topic?}

- Staphylococcal FnbpA and ClfA are major surface molecules contributing to endothelial cell adhesion.

- FnbpA binds via fibronectin retained on the endothelial $\alpha 5 \beta 1$ integrin and ClfA to the $\gamma$-chain of fibrinogen, allowing its binding to endothelial cell and platelet integrin receptors.

- Furthermore, S. aureus ClfA adheres to endothelial vWF via the staphylococcal vWbp in a shear stressdependent manner.

\section{What does this paper add?}

- In the absence of plasma and under shear stress, lactococci expressing FnbpA and ClfA interact comparably with endothelial cells via the pathways described for S. aureus-endothelial cell adhesion, as EC-bound fibronectin, EC-bound fibrinogen and EC-bound vWF.

- L. lactis-clfA also binds to fibrin via its $\gamma$-chain. In the presence of plasma, the efficiency of FnbpA is reduced by fourfold, whereas that of ClfA towards EC-bound vWF is rapidly reduced by $>$ threefold which is the result of vWF-hydrolysis by ADAMTS13.

- Hence, in plasma containing high fibrinogen concentrations, ClfA is a highly relevant bacterial adhesin due to its interactions with EC-bound fibrinogen.

\section{Authors' Contributions}

M.H., R.H., B.D., T.V., L.L., T.R.V. and P.V. designed the research. J.C. and B.D. performed the experiments and analysed the data. J.C., B.D., M.H. and R.H. wrote the manuscript. P.M. and J.M.E. designed the research and provided the L. lactis strains. B.D. performed all work of the revision.

\section{Funding}

This study was sponsored by a grant of the Research Fund KU Leuven (OT/14/097) and by the Programmafinanciering KU Leuven (PF/10/014). T.R.V. was a postdoctoral fellow of the FWO Research Foundation-Flanders (Belgium; Grant Number - 12K0916N) and R.H. is supported by the Clinical Research Fund of UZ Leuven.

\section{Conflict of Interest}

P.V. reports grants and personal fees from BoehringerIngelheim, Bayer, Daiichi Sankyo and Pfizer, outside the area of this work. All other authors have no conflict of interest and nothing to disclose with regard to commercial support. 


\section{Acknowledgments}

We thank K. Cludts, S. Van Kerckhoven and M. BezulskaDitkowska for their skilful technical assistance. We also thank O. Schneewind, Department of Microbiology, University of Chicago, for providing vWbp purified from $E$. coli BL21. We are grateful to the co-workers of the Department of Gynaecology at UZ Leuven for providing human umbilical cords.

\section{References}

1 Lowy FD. Staphylococcus aureus infections. N Engl J Med 1998; 339(08):520-532

2 Fitzgerald JR, Loughman A, Keane F, et al. Fibronectin-binding proteins of Staphylococcus aureus mediate activation of human platelets via fibrinogen and fibronectin bridges to integrin GPIIb/ IIIa and IgG binding to the FcgammaRIIa receptor. Mol Microbiol 2006;59(01):212-230

3 Heying R, van de Gevel J, Que YA, Piroth L, Moreillon P, Beekhuizen H. Contribution of (sub)domains of Staphylococcus aureus fibronectin-binding protein to the proinflammatory and procoagulant response of human vascular endothelial cells. Thromb Haemost 2009;101(03):495-504

4 Massey RC, Kantzanou MN, Fowler T, et al. Fibronectin-binding protein A of Staphylococcus aureus has multiple, substituting, binding regions that mediate adherence to fibronectin and invasion of endothelial cells. Cell Microbiol 2001;3(12):839-851

5 Piroth L, Que YA, Widmer E, et al. The fibrinogen- and fibronectinbinding domains of Staphylococcus aureus fibronectin-binding protein A synergistically promote endothelial invasion and experimental endocarditis. Infect Immun 2008;76(08):3824-3831

6 Wann ER, Gurusiddappa S, Hook M. The fibronectin-binding MSCRAMM FnbpA of Staphylococcus aureus is a bifunctional protein that also binds to fibrinogen. J Biol Chem 2000;275(18): 13863-13871

7 Patti JM, Allen BL, McGavin MJ, Höök M. MSCRAMM-mediated adherence of microorganisms to host tissues. Annu Rev Microbiol 1994;48:585-617

8 McDevitt D, Nanavaty T, House-Pompeo K, et al. Characterization of the interaction between the Staphylococcus aureus clumping factor (ClfA) and fibrinogen. Eur J Biochem 1997;247(01):416-424

9 Siboo IR, Cheung AL, Bayer AS, Sullam PM. Clumping factor A mediates binding of Staphylococcus aureus to human platelets. Infect Immun 2001;69(05):3120-3127

10 Allgayer S, Rosenbach G, Tavares CA, Polido WD. Periodontal ligament distraction: esthetics and occlusal stability at the 2-year follow-up. Am J Orthod Dentofacial Orthop 2013;143(04):535-546

11 Foster TJ. The remarkably multifunctional fibronectin binding proteins of Staphylococcus aureus. Eur J Clin Microbiol Infect Dis 2016;35(12):1923-1931

12 Mazmanian SK, Liu G, Jensen ER, Lenoy E, Schneewind O. Staphylococcus aureus sortase mutants defective in the display of surface proteins and in the pathogenesis of animal infections. Proc Natl Acad Sci U S A 2000;97(10):5510-5515

13 Mazmanian SK, Liu G, Ton-That H, Schneewind O. Staphylococcus aureus sortase, an enzyme that anchors surface proteins to the cell wall. Science 1999;285(5428):760-763

14 Schneewind O, Mihaylova-Petkov D, Model P. Cell wall sorting signals in surface proteins of gram-positive bacteria. EMBO J 1993;12(12):4803-4811

15 Ton-That H, Liu G, Mazmanian SK, Faull KF, Schneewind O. Purification and characterization of sortase, the transpeptidase that cleaves surface proteins of Staphylococcus aureus at the LPXTG motif. Proc Natl Acad Sci U S A 1999;96(22):12424-12429

16 Pappelbaum KI, Gorzelanny C, Grässle S, et al. Ultralarge von Willebrand factor fibers mediate luminal Staphylococcus aureus adhesion to an intact endothelial cell layer under shear stress. Circulation 2013;128(01):50-59

17 Claes J, Vanassche T, Peetermans M, et al. Adhesion of Staphylococcus aureus to the vessel wall under flow is mediated by von Willebrand factor-binding protein. Blood 2014;124(10):1669-1676

18 Schwarz-Linek U, Werner JM, Pickford AR, et al. Pathogenic bacteria attach to human fibronectin through a tandem betazipper. Nature 2003;423(6936):177-181

19 Que YA, Haefliger JA, Piroth L, et al. Fibrinogen and fibronectin binding cooperate for valve infection and invasion in Staphylococcus aureus experimental endocarditis. J Exp Med 2005;201 (10):1627-1635

20 Sinha B, Francois P, Que YA, et al. Heterologously expressed Staphylococcus aureus fibronectin-binding proteins are sufficient for invasion of host cells. Infect Immun 2000;68(12):6871-6878

21 Strindhall J, Lindgren PE, Löfgren S, Kihlström E. Variations among clinical isolates of Staphylococcus aureus to induce expression of E-selectin and ICAM-1 in human endothelial cells. FEMS Immunol Med Microbiol 2002;32(03):227-235

22 Peacock SJ, Foster TJ, Cameron BJ, Berendt AR. Bacterial fibronectin-binding proteins and endothelial cell surface fibronectin mediate adherence of Staphylococcus aureus to resting human endothelial cells. Microbiology 1999;145(Pt 12):3477-3486

23 Barczyk M, Carracedo S, Gullberg D. Integrins. Cell Tissue Res 2010;339(01):269-280

24 Que YA, François P, Haefliger JA, Entenza JM, Vaudaux P, Moreillon P. Reassessing the role of Staphylococcus aureus clumping factor and fibronectin-binding protein by expression in Lactococcus lactis. Infect Immun 2001;69(10):6296-6302

25 Claes J, Liesenborghs L, Peetermans M, et al. Clumping factor A, von Willebrand factor-binding protein and von Willebrand factor anchor Staphylococcus aureus to the vessel wall. J Thromb Haemost 2017;15(05):1009-1019

26 Que YA, Haefliger JA, Francioli P, Moreillon P. Expression of Staphylococcus aureus clumping factor A in Lactococcus lactis subsp. cremoris using a new shuttle vector. Infect Immun 2000;68 (06):3516-3522

27 Claes J, Liesenborghs L, Lox M, Verhamme P, Vanassche T, Peetermans $M$. In vitro and in vivo model to study bacterial adhesion to the vessel wall under flow conditions. J Vis Exp 2015;(100):e52862

28 Theilmeier G, Lenaerts T, Remacle C, Collen D, Vermylen J, Hoylaerts MF. Circulating activated platelets assist THP-1 monocytoid/endothelial cell interaction under shear stress. Blood 1999;94(08):2725-2734

29 Entenza JM, Moreillon P, Senn MM, et al. Role of sigmaB in the expression of Staphylococcus aureus cell wall adhesins ClfA and FnbA and contribution to infectivity in a rat model of experimental endocarditis. Infect Immun 2005;73(02):990-998

30 Vanassche T, Kauskot A, Verhaegen J, et al. Fibrin formation by staphylothrombin facilitates Staphylococcus aureus-induced platelet aggregation. Thromb Haemost 2012;107(06):1107-1121

31 McAdow M, Missiakas DM, Schneewind O. Staphylococcus aureus secretes coagulase and von Willebrand factor binding protein to modify the coagulation cascade and establish host infections. J Innate Immun 2012;4(02):141-148

32 Veltrop MH, Beekhuizen H, Thompson J. Bacterial species- and strain-dependent induction of tissue factor in human vascular endothelial cells. Infect Immun 1999;67(11):6130-6138

33 Kerrigan SW, Clarke N, Loughman A, Meade G, Foster TJ, Cox D. Molecular basis for Staphylococcus aureus-mediated platelet aggregate formation under arterial shear in vitro. Arterioscler Thromb Vasc Biol 2008;28(02):335-340

34 Niemann S, Spehr N, Van Aken H, et al. Soluble fibrin is the main mediator of Staphylococcus aureus adhesion to platelets. Circulation 2004;110(02):193-200

35 Bjerketorp J, Jacobsson K, Frykberg L. The von Willebrand factorbinding protein (vWbp) of Staphylococcus aureus is a coagulase. FEMS Microbiol Lett 2004;234(02):309-314 
36 Thomer L, Schneewind O, Missiakas D. Multiple ligands of von Willebrand factor-binding protein (vWbp) promote Staphylococcus aureus clot formation in human plasma. J Biol Chem 2013;288 (39):28283-28292

37 Friedrich R, Panizzi P, Fuentes-Prior P, et al. Staphylocoagulase is a prototype for the mechanism of cofactor-induced zymogen activation. Nature 2003;425(6957):535-539

38 Kroh HK, Panizzi P, Bock PE. Von Willebrand factor-binding protein is a hysteretic conformational activator of prothrombin. Proc Natl Acad Sci U S A 2009;106(19):7786-7791
39 Schneider H, Vogt M, Boekenkamp R, et al. Melody transcatheter valve: Histopathology and clinical implications of nine explanted devices. Int J Cardiol 2015;189:124-131

40 Kremer Hovinga JA, Zeerleder S, Kessler P, et al. ADAMTS-13, von Willebrand factor and related parameters in severe sepsis and septic shock. J Thromb Haemost 2007;5(11):2284-2290

41 Aibar J, Castro P, Espinosa G, et al. ADAMTS-13 in critically ill patients with septic syndromes and noninfectious systemic inflammatory response syndrome. Shock 2015;43(06): $556-562$ 